\title{
Inferior vena cava thrombosis as a complication of femoral vein catheterisation
}

\author{
A Shefler, J Gillis, A Lam, A J O'Connell, D Schell, A Lammi
}

\begin{abstract}
Indwelling femoral venous catheters were prospectively studied by ultrasonography to define the frequency and evolution of inferior vena cava (IVC) thrombosis. IVC thrombosis was identified in six of 56 catheters ( 54 children). Only one patient with a positive ultrasound scan had clinical signs of thrombosis. All children with IVC thrombosis had had catheters in place for over six days. It is recommended that either the femoral central venous catheters are routinely changed at six days or ultrasound studies are routinely performed twice a week in all patients with catheters in situ for six or more days and that the catheter is removed immediately if evidence of thrombosis appears.

(Arch Dis Child 1995; 72: 343-345)
\end{abstract}

Keywords: central line, femoral vein, inferior vena cava thrombosis.

The femoral vein has become an increasingly preferred site for central venous access in paediatric intensive care patients. Catheterisation via this route is associated with a low incidence of complications and avoids the risk of injury to the structures in the neck and thoracic cavity. ${ }^{1-3}$ As with all central venous catheterisation procedures, there remains a recognised risk of venous thrombosis. ${ }^{4-6}$ Inferior vena cava (IVC) thrombosis has been reported with femoral venous catheters in both children and adults, ${ }^{7-9}$ but the incidence and evolution of this complication in paediatric intensive care practice is not well documented. Ultrasonography is a rapid, portable, effective means of evaluating the presence of thrombus in the IVC. ${ }^{10}$ Using sequential ultrasound data, the present descriptive study attempted to define the frequency and evolution of IVC thrombosis in children with indwelling femoral venous catheters.

Children's Hospital, Pyrmont Bridge Road, Camperdown, NSW, 2050 Australia, Intensive Care Unit A Shefler $J$ Gillis

A J O'Connell

D Schell

Department of Radiology

A Lam

Department of

Haematology

A Lammi

Correspondence to: Dr Gillis.

Accepted 3 January 1995

\section{Patients and methods}

Data were collected prospectively from all patients undergoing femoral venous catheterisation in the general intensive care unit (which excludes postoperative cardiac patients) from August 1991 to October 1992. The study was approved by the hospital ethics committee and written consent was obtained before enrolling each patient. Children were excluded if catheters were in place for fewer than 48 hours or used for haemodialysis. Data regarding age, diagnosis, duration of catheterisation, and patient. Blood samples were taken at the time of catheter insertion for coagulation profiles and antithrombin III measurements.

\section{CATHETER INSERTION AND USE}

Catheters were placed for central venous pressure monitoring as well as administration of fluids, total parenteral nutrition, medications, and blood products. All femoral venous catheters were placed percutaneously according to the Seldinger technique by residents or consultants on the intensive care unit. Aseptic technique included surgical hand scrub, sterile gown and gloves, masks, and sterile drapes for all procedures. All sites were cleansed with a $0.5 \%$ chlorhexidine in $70 \%$ ethanol solution. A sterile transparent adhesive dressing was applied. Multilumen polyurethane catheters (Arrow) were used in all cases. Catheter diameter varied according to the patient size. All catheter tip positions were confirmed on plain radiographs immediately after insertion. Tips were positioned to within $5 \mathrm{~cm}$ of the right atrium. Catheter lumina used for haemodynamic monitoring were infused with a heparinised $(0.5$ units $/ \mathrm{ml})$ dextrose or normal saline solution. Unused ('locked') lumina were flushed every six hours with a concentrated heparinised solution (10 units $/ \mathrm{ml}$ ).

\section{ULTRASOUND EXAMINATIONS}

All patients underwent ultrasound examination within the first three days after catheter insertion and every two to four days thereafter until removal of the catheter. Wherever possible, patients were studied to within 24 hours of catheter removal. Scans were performed with an Ultramark 4 real time scanner with a 5 or $7.5 \mathrm{mHz}$ transducer and Acuson 128XP scanner with colour Doppler if a thrombus was detected. haemodynamic status were recorded for each

\section{Results}

Over the 14 month study period, 61 femoral venous catheters were inserted in 59 patients who fulfilled the selection criteria. Five patients were not studied due to logistic reasons or refusal to participate in the study. Thus, 56 catheters in 54 patients were available for analysis. There were 32 boys and 22 girls; mean age at the age of insertion was $37 \cdot 6$ months (range $0.5-192$ months). There were a wide range of diagnoses, and the overall mortality was $6 \%$. Catheters were in place for a mean (and median) duration of nine days. cally in six patients, that is $10 \cdot 7 \%$ (six of 56 of
IVC thrombosis was identified sonographi- 
catheters placed). In all six patients, thrombus was detected by ultrasound on or after the eighth day of catheterisation. Five of the six patients had had a negative ultrasound 72 hours previously. Thrombi were detected on days $8,8,10,20$, and 20 . The sixth patient was entered later in the study and the first ultrasound was not performed until the 13th day after insertion when a thrombus was detected. The time of first appearance of thrombus in this patient is therefore not known. Only one patient with a positive ultrasound had clinical signs of thrombosis. No clinical signs were detected in the remainder of the study population.

The diagnoses of those children with thrombus were: pneumocystis pneumonia in a child on immunosuppressives for renal disease; one patient with a near drowning episode and severe adult respiratory distress syndrome; one patient with staphylococcal pneumonia; one patient with encephalopathy of unknown origin; one patient with haemophilus meningitis; and one patient with a meningoencephalitis of probable viral cause. These clinical pictures did not differ in any significant way from the group without thrombus. The diagnoses in these children included 18 with respiratory pathology including pneumonia; four with meningitis and/or encephalitis; two with near drowning; five with sepsis including two episodes associated with immunosuppression; seven with various central nervous system disorders including trauma and seizures; four children with metabolic or liver disorders; and eight with various miscellaneous diagnoses.

The mean age of those children with and those without thrombus was 37.6 months. Altogether $83 \%(5 / 6)$ of those children with a thrombus had received inotropic medication at some point while the catheter was in situ whereas only $50 \%(25 / 50)$ of those without a thrombus received similar treatment, and the same number of children with thrombosis (5/6) had prolonged coagulation profiles while in the non-thrombosis group only $48 \%(24 / 50)$ had prolonged coagulation profiles. When antithrombin III was measured, two out of five patients $(40 \%)$ in the thrombosis group and 17 of 45 patients $(38 \%)$ in the non-thrombosis group had low concentrations.

In each case of IVC thrombosis, the femoral catheter was removed as soon as the diagnosis was made by ultrasound. Five of six cases were followed up with sonograms until complete resolution of the thrombus (five to 31 days). The sixth patient was lost to follow up. One patient was treated with intravenous heparin for a period of 18 days (time to resolution 31 days); the other five children received no specific treatment other than catheter removal.

\section{Discussion}

The use of femoral central venous catheters in the paediatric intensive care unit is increasing. Although the low complication rate compares favourably with non-femoral routes, ${ }^{11}$ the occurrence of thrombosis in the IVC and large pelvic veins has only been recorded once in a brief report showing a $36 \%$ incidence of femoral vein thrombosis. ${ }^{12}$ In our study IVC thrombosis was demonstrated in $10.7 \%$ of patients using sequential ultrasound examinations. Large vessel thrombi are usually clinically silent ${ }^{13}$ and the paucity of signs in our group emphasises the need for systematic surveillance to enhance early detection. While venography has been a useful imaging modality in the past, ${ }^{13} 14$ ultrasound has the advantage of being a safe, non-invasive means of studying the IVC, and lends itself well to assessment of critically ill patients immobilised in the intensive care unit. The ability of ultrasound to visualise clearly the IVC and identify the presence of intraluminal thrombus has been well documented. ${ }^{101516}$ Furthermore, in the evaluation of thrombi in the IVC, ultrasound may have an additional advantage over venography in more clearly delineating the cephalad extent of thrombotic occlusion. ${ }^{15}$

Previous data have suggested a relationship between the length of time a catheter is in situ and thrombotic complications. ${ }^{912} 1417$ In our study, no thrombi were identified on ultrasound before eight days of catheter use and it seems reasonable to assume that patients with femoral catheters in for fewer than seven days are at lower risk.

The low incidence of IVC thrombosis in our study precludes clear definition of specific at risk patients. However, the majority of our cases with positive ultrasound findings received inotropes. This supports previous observations that dehydration and low perfusion may predispose to thrombus formation in association with intravascular catheters. Decreased antithrombin III concentrations in the majority of patients in this setting suggest that this test is unlikely to be useful as a predictor of increased risk.

The risk of pulmonary embolus in the presence of IVC thrombosis in adults has been well described. ${ }^{1819}$ No patients in our study exhibited obvious signs of pulmonary embolus but many were intubated and artificially ventilated for severe pulmonary disease. The risk of this potentially lethal complication associated with catheter related thrombus in the IVC is unknown, although pulmonary embolism has been reported in association with femoral catheterisation. ${ }^{9} 20$

Guidelines for the management of IVC thrombosis in the paediatric patient in this setting are lacking. Anticoagulation was instituted in one patient with extensive thrombosis reflected by the appearance of clinical signs and the slow resolution of the thrombus. It is difficult to make firm recommendations but appropriate management would appear to consist of removal of the catheter, institution of anticoagulation, and regular follow up ultrasonography.

In summary, we were able to detect the presence of thrombus in the IVC of $10 \%$ of critically ill patients catheterised via the femoral route using serial bedside ultrasound examinations. While it is possible that a subgroup of patients with more severe illness may be at higher risk of developing this complication, we 
were unable to define adequately the risk factors. We found a relationship between thrombus formation and duration of catheter use and recommend routine ultrasound study of all patients with femoral central venous catheters in situ for six or more days. Scans should be performed twice a week thereafter for the duration of catheter use. An alternative strategy would be to change routinely the site of the central venous catheter at six days. Although the embolic risk is unknown, we advise removal of the catheter if a thrombus is identified, anticoagulation, and twice weekly ultrasound follow up until complete recanalisation.

1 Canter RK, Zimmerman J, Strauss RH. Central venous catheter insertion by the femoral vein: safety and effectiveness for the pediatric patient. Pediatrics 1986; 77: 842.

2 Williams JF, Seneff MG, Friedman BC, et al. Use of femoral catheters in critically ill adults: prospective study. Crit Care Med 1991; 19: 550

3 Getzen LC, Pollack EW. Short-term femoral vein catheterisation. A safe alternative venous access? Am $\mathcal{F}$ Surg 1979; 138: 875 .

4 Lindblad B. Thromboembolic complications and central venous catheters. Lancet 1982; ii: 936.

5 Williams EC. Catheter-related thrombosis. Clin Cardiol 1990; 13: 34 .

6 Ahmed N. Thrombosis after central venous cannulation. Med $\mathcal{A}$ Aust 1976; i: 217.

7 Riggs T, Hirschfeld W, Borkat G, et al. Inferior vena cava obstruction secondary to indwelling venous catheters: two
ond cases. Pediatrics 1976; 58: 446 .
8 Gouge $\mathrm{MD}$, Paulson WD, Moore J. Inferior vena cava thrombosis due to an indwelling hemodialysis catheter. Am 7 Kidney Dis 1988; 11: 515.

9 Bansmer $\mathrm{G}$, Keith $\mathrm{D}$, Tesluk $\mathrm{H}$. Complications following use of indwelling catheters of the inferior cava. $¥ A M A$ 1958; 167: 1606 .

10 Goiney R. Ultrasound imaging of inferior vena cava thrombosis. F Ultrasound Med 1985; 4: 387

11 Stenzel JP, Green TP, Fuhrman BP, et al. Percutaneous femoral venous catheterisations: a prospective study of complications. F Pediatr 1989; 114: 41

12 Krafte-Jacobs B, Sivitc C, Mejia R, Pollack M. Catheter related femoral venous thrombosis in critically ill children. Crit Care Med 1994; 22: A159.

13 Moore RA, McNicholas KW, Naidech $\mathrm{H}$, et al. Clinically silent venous thrombosis following internal and external centra venous cannulation in pediatric cardiac patients. Anesthesiology 1985; 62: 640 .

14 Peters WR, Bush WH, McIntyre RD, et al. The development of fibrin sheath on indwelling venous catheters. Surg Gynecol Obstet 1973; 137: 43 .

15 Park JH, Lee JB, Han MC, et al. Somographic evaluation of inferior vena caval obstruction: correlative study with vena inferior vena caval obstruction: cort

16 Sonnenfeld $M$, Finberg HJ. Ultrasonographic diagnosis of incomplete inferior vena caval thrombosis secondary to periphlebitis: the importance of a complete survey examination. Radiology 1980; 137: 743.

17 Hoshal VL, Ause RG, Hoskins PA. Fibrin sleeve formation on indwelling subclavian central venous catheters. Arch Surg 1971; 102: 353.

18 Radomski JS, Jarrell BE, Carabasi RA, et al. Risk of pulmonary embolus with inferior vena cava thrombosis. $\mathrm{Am}$ Surg 1987; 53: 97.

19 Girard MD, Hauuy MP, Mussett D, et al. Acute inferior vena cava thrombosis. Early results of heparin therapy. Chest 1989; 95: 284.

20 Lynn KL, Maling TMJ. A major pulmonary embolus as a complication of femoral vein catheterisation. $\mathrm{Br} \mathcal{f}$ Radiol 1977; 50: 667 . 